\title{
Long-term recurrence-free survival after hepatic resection for metachronous gastric cancer liver metastases
}

\author{
Bert Peeters $^{1 *}$, Jeroen Kerstens ${ }^{1 *}$, Pol Specenier ${ }^{1,2}$ \\ ${ }^{1}$ University of Antwerp, Faculty of Medicine and Health Sciences, 2650 Edegem, Belgium; ${ }^{2}$ Department of Oncology, Antwerp University Hospital, \\ 2650 Edegem, Belgium \\ *These authors contributed equally to this article. \\ Correspondence to: Pol Specenier, MD, PhD. Department of Oncology, Antwerp University Hospital, Wilrijkstraat 10, 2650 Edegem, Belgium. \\ Email: pol.specenier@uza.be.
}

\begin{abstract}
We present the case of a man who underwent total gastrectomy and splenectomy at the age of 60 for stage IIIa adenocarcinoma of the stomach. Twenty-two months after surgery abdominal CT scan showed two hepatic metastases in segments V and VI. Because of further progression under palliative chemotherapy and reduced tolerance for this treatment, partial hepatectomy of segments V-VI was performed. Currently 72 years old, he is completely symptom-free without evidence for tumor recurrence. This report shows that long-term recurrence-free survival after hepatic resection for gastric cancer liver metastases (GCLM) is possible even in the presence of several unfavorable prognostic factors.
\end{abstract}

Keywords: Gastric cancer; liver metastasis; hepatectomy

Submitted Mar 03, 2016. Accepted for publication Apr 07, 2016.

doi: $10.21037 /$ tcr.2016.07.04

View this article at: http://dx.doi.org/10.21037/tcr.2016.07.04

\section{Introduction}

Gastric cancer is the fifth most common malignancy and the third leading cause of cancer death in both sexes worldwide, with 952,000 new cases $(6.8 \%$ of the total) and 723,000 deaths $(8.8 \%$ of the total) in 2012 (1). The liver is one of the most common sites of metastatic spread from malignancies of the stomach, with hepatic metastases [gastric cancer liver metastases (GCLM)] occurring in up to $50 \%$ of the patients (2). Three histological subtypes of gastric cancer are likely to give rise to GCLM: differentiated adenocarcinoma, poorly differentiated adenocarcinoma, and the rarer hepatoid carcinoma (2). Metastatic gastric cancer has a dismal prognosis with a median survival of 6 months if untreated and 7-15 months with palliative chemotherapy (3). Survival data for patients with GCLM without further extrahepatic metastases are scarce, with one study reporting 5 -year survival of $1.7 \%$ for patients with GCLM alone that were treated with systemic therapy (4). In this report we present a rare case of longterm recurrence-free survival of a patient with differentiated adenocarcinoma of the stomach who received curative surgery with resection of two metachronous GCLM.

\section{Case presentation}

In December 2003, a 60-year-old Caucasian man underwent total gastrectomy and splenectomy for a moderately differentiated intestinal-type pT3N1M0 (stage IIIa) adenocarcinoma of the gastric cardia. In October 2005, twenty-two months after surgery, an abdominal CT scan was performed after routine blood tests had shown chronically elevated alkaline phosphatase (126-137 U/L; normal range, 36-95 U/L) with progressively rising tumor marker carcinoembryonic antigen (CEA) (from 36 to $195 \mathrm{ng} / \mathrm{mL}$; normal range, $<3.4 \mathrm{ng} / \mathrm{mL}$ for non-smokers) over the last eight months. This investigation revealed two hepatic metastases in segments V and VI (largest lesion: $3.2 \times 2.5 \mathrm{~cm}^{2}$ ) along with multiple hepatic biliary cysts. As the patient was completely symptom-free at this moment, we initially opted for a strategy of watchful waiting, but at re-evaluation three months later there was evidence of progression with confluence of the hepatic metastases on CT scan and 
further elevation of CEA $(274 \mathrm{ng} / \mathrm{mL})$.

In March 2006, we started palliative chemotherapy with 5 -fluorouracil ( 5 -FU) $1,000 \mathrm{mg} / \mathrm{m}^{2} /$ day for 5 days and cisplatin $100 \mathrm{mg} / \mathrm{m}^{2}$ on day 1 , every 3 weeks. Cisplatin was substituted by carboplatin after one cycle because of mild high-frequency hearing loss. Re-evaluations after two and four cycles respectively showed a partial response accompanied by a decrease in CEA. However, after six cycles of chemotherapy, progression of the hepatic metastases was observed on CT scan and CEA started to increase again. PET scan in July 2006 showed increased FDG uptake in segment V-VI of the liver but no other hepatic metastases. Because of reduced tolerance for the chemotherapy treatment the patient was offered surgical resection of the metastases. Additional preoperative imaging with MRI of the liver confirmed the presence of a voluminous hepatic metastasis in segment VI $\left(9 \times 5.8 \mathrm{~cm}^{2}\right)$ and a smaller satellite metastasis located caudally in segment $\mathrm{V}$, but revealed no further active metastatic processes in the other segments. The patient underwent partial hepatectomy of segments V-VI and removal of two lymph nodes, with anatomopathological examination confirming R0 resection. He quickly and fully recovered with complete normalization of CEA and remained in follow-up at our department with clinical examination, routine blood tests and control of CEA every three months and abdominal CT scan every year. Currently 72 years old, he is completely symptomfree without clinical, biochemical or radiological evidence for tumor recurrence or metastases nine years after partial hepatectomy and almost twelve years after resection of the gastric primary.

\section{Discussion}

The position of hepatic resection in the context of GCLM remains controversial and is at present by no means standard of care. This is in part due to the fact that very few patients with GCLM are eligible for curative surgery because of the presence of multiple, scattered, bilobar liver lesions or incurable simultaneous factors such as peritoneal dissemination, widespread lymph nodal metastases, or direct invasion to adjacent structures (3). However, when dealing with liver-confined metastatic gastric cancer, R0 resection of GCLM is the only therapeutic option that can be undertaken with a curative intent. Over the last decade, several retrospective cohort studies have looked at survival after hepatic resection for GCLM compared to other treatment options and have tried to identify favourable prognostic factors that may permit selection of good candidates for this procedure.

A first systematic review of 19 studies with a total of 436 patients reported median and 5-year overall survival after surgery for GCLM ranging from 9 to 34 months (median: 17 months) and 0-60\% (median: $26.5 \%$ ), respectively (5). A second, more recent systematic review and meta-analysis of 23 studies comprising 870 patients found that median and 5 -year overall survival ranged from 8.8 to 48 months and $0-60 \%$, respectively, with a pooled value of 22 months and $24 \%$ (30\% for metachronous lesions) (6).

As for prognostic factors, synchronous presentation of GCLM, short recurrence-free interval from primary resection ( $<1$ year), the presence of multiple or larger GCLM, bilobar disease, and primary tumor factors such as increased depth of invasion (T-stage), higher degree of lymphatic and venous invasion and moderate or poorly differentiated histological grade were al found to be associated with worse prognosis by some of the studies reviewed by Kerkar et al. (5). However, the authors note that based on their analysis, no definitive conclusions can be made regarding negative prognostic factors because no factor was found to be consistently statistically significant across studies (5). The second systematic review by Petrelli et al. found that only patients with larger size and number of GCLM were found in at least three studies to carry a worse prognosis (6).

Recently, a working group installed by the guidelines committee of the Japan Gastric Cancer Association reviewed the literature to revisit the treatment of potentially resectable GCLM and listed smaller size (diameter <4-6 cm) and number of GCLM, unilobular distribution, capsular formation, and status of the gastric primary (absence of serosal or lymphatic invasion, lower clinical stage) as potentially relevant favourable prognostic factors (7).

Long-term survivors in a disease as aggressive as metastatic gastric cancer are rare. Our case is even more remarkable because our patient managed to survive for almost twelve years now despite several factors that are reported to possibly adversely affecting prognosis: the presence of more than one hepatic lesion, the large size of one of the lesions $\left(9 \times 5.8 \mathrm{~cm}^{2}\right)$ and the high clinical stage and moderate differentiation of the gastric primary (pT3N1M0: stage IIIa). In the systematic review by Kerkar et al., detailed analyses of data for a total of seven patients who survived for $\geq 10$ years showed that all of them had only a single lesion resected, four of six presented with a welldifferentiated histological grade and four of five patients had 
a T1-T2 primary lesion (5). Furthermore, the progression of the metastases under systemic chemotherapy can also be considered as a sign of the aggressive nature of the tumor (7). On the other hand, factors contributing to the successful outcome in this case presumably are the relatively long recurrence-free interval after gastrectomy (22 months), the absence of extrahepatic disease and the good overall clinical condition of the patient.

\section{Conclusions}

In conclusion, hepatic resection for GCLM seems to be associated with an acceptable 5-year survival in selected patients, while the precise factors on which to base this selection are still under debate. Most literature seems to indicate that patients with a small number of GCLM and without extrahepatic disease, could be offered hepatic resection if this is technically feasible and safe (6). Although guidance in this field should come from properly designed randomized controlled trials, our case report expands the literature on the subject and shows that surgery with curative intent can successfully extend survival even in patients with multiple lesions, lesions with a diameter $>5 \mathrm{~cm}$ and a high-stage primary tumor.

\section{Acknowledgements}

None.

\section{Footnote}

Conflicts of Interest: The authors have no conflicts of interest

Cite this article as: Peeters B, Kerstens J, Specenier P. Longterm recurrence-free survival after hepatic resection for metachronous gastric cancer liver metastases. Transl Cancer Res 2016;5(4):497-499. doi: 10.21037/tcr.2016.07.04 to declare.

Informed Consent: Written informed consent was obtained from the patient for publication of this case report.

\section{References}

1. Ferlay J, Soerjomataram I, Ervik M, et al. GLOBOCAN 2012 v1. 0, Cancer Incidence and Mortality Worldwide: IARC CancerBase No. 11, 2013. Available online: http:// globocan.iarc.fr/Default.aspx

2. Kakeji Y, Morita M, Maehara Y. Strategies for treating liver metastasis from gastric cancer. Surg Today 2010;40:287-94.

3. Romano F, Garancini M, Uggeri F, et al. Surgical treatment of liver metastases of gastric cancer: state of the art. World J Surg Oncol 2012;10:157.

4. Yoshida M, Ohtsu A, Boku N, et al. Long-term survival and prognostic factors in patients with metastatic gastric cancers treated with chemotherapy in the Japan Clinical Oncology Group (JCOG) study. Jpn J Clin Oncol 2004;34:654-9.

5. Kerkar SP, Kemp CD, Avital I. Liver resections in metastatic gastric cancer. HPB (Oxford) 2010;12:589-96.

6. Petrelli F, Coinu A, Cabiddu M, et al. Hepatic resection for gastric cancer liver metastases: A systematic review and meta-analysis. J Surg Oncol 2015;111:1021-7.

7. Kodera Y, Fujitani K, Fukushima N, et al. Surgical resection of hepatic metastasis from gastric cancer: a review and new recommendation in the Japanese gastric cancer treatment guidelines. Gastric Cancer 2014;17:206-12. 